\title{
PELVIC CHONDROSARCOMA - THE ROLE OF THE BIOPSY AND THE CORRELATION BETWEEN THE HISTOPATHOLOGICAL BIOPTIC DIAGNOSIS AND THE EXCISIONAL ONE
}

doi: 10.2478/rojost-2018-0079

\author{
R. Popescu ${ }^{12}$, Șt. Cuculici ${ }^{13}$, Șt. Cristea ${ }^{13}$ \\ ${ }^{1 "}$ Carol Davila" University of Medicine and Pharmacy, Bucharest, Romania \\ "Foişor" Hospital of Orthopaedics, Trauma and OsteoarticularTB, Bucharest, Romania \\ 3"Sf. Pantelimon" Emergency Hospital, \\ Bucharest, Romania
}

Chondrosarcoma is one of the most frequent malignant bone tumors. We included in the initial study population 110 patients with pelvis biopsies and the histopathological result of chondrosarcoma. We excluded the cases with incomplete data. Thirty-five in block resections were included in the final study population. In the rest of the cases, the surgery was not made because of many considerations (multiple metastases, patient's refusal, and surgical contraindications). In three cases, discrepancies were noted between the grade of malignancy found after biopsy and the one confirmed through the surgical excision. This corresponds to a precision rate of $91,42 \%$ (32/35) in the confirmation of chondrosarcoma. The accuracy of the histopathological diagnosis between low grade chondrosarcoma and high grade chondrosarcoma was of $94,27 \%(33 / 35)$. We concluded that the image-guided biopsy is useful in determining the grade of malignancy of the chondrosarcoma and in the planning of the subsequent treatment. A multidisciplinary team (orthopaedic surgeon, radiologist, and pathologist) is needed to improve the diagnosis of the pelvic chondrosarcoma. Keywords: chondrosarcoma, biopsy, anatomopathological diagnosis, pelvis 